\title{
Effective patient safety education for novice RNs: A systematic review
}

\author{
MaryAnn Niemeyer* \\ Christian Hospital Center for Training \& Development, Saint Louis, Missouri, United States
}

Received: August 29, 2017

Accepted: November 6, $2017 \quad$ Online Published: November 20, 2017

DOI: $10.5430 /$ jnep.v8n3p103

URL: https://doi.org/10.5430/jnep.v8n3p103

\begin{abstract}
Background and objective: The need is great for identifying effective evidence-based strategies that focus on increasing novice $\mathrm{RN}$ confidence for the application of skills used to care for patients safely. The purpose of this systematic review is to explore effective continuing education strategies that target novice RNs' professional development, enhance clinical confidence, and focus on patient safety.

Methods: The EBSCOhost database search was set to find recently published papers within the last ten years, sorted by relevance from January 2007 through August 2017. This search yielded twelve studies deemed eligible for inclusion by the databases CINAHL, Communication \& Mass Media Complete, Education Full Text (H.W. Wilson), Health \& Wellness Resource Center, and Science Direct. Commonalities and distinguishing features among the strategies are examined.

Results: This systematic review identified 12 articles that describe effective training strategies aimed at improving novice RNs' clinical practice confidence and skill. A thematic analysis of the data was used to systematically gain knowledge about strategies used to educate novice RNs working in the hospital setting. The majority of strategies employed a number of different types of simulation and reported varying degrees of success for improving novice RN ability to care for patients safely. Simulation, virtual reality, preceptored clinical experiences, and interdisciplinary experiences were found to be effective education strategies enhancing novice RN's skill for providing safe care. Didactic instruction had positive results, but was not as effective as simulation for novice RNs learning safe patient care. Finally, written instruction was not as effective as simulation, and hard copy supplements provided no added value to novice RNs learning safe patient care.

Conclusions: Findings from this review are foundational to address calls from the Institute of Medicine (IOM) and the National League for Nursing (NLN) to reform and support post-graduate nursing education. The development of novel education and training targeting novice RNs in the hospital setting is essential, but more research is needed to enhance safe patient care.
\end{abstract}

Key Words: Confidence, Competence, Continuing education, Novice RN, Medical error, Patient safety, Teaching strategy, Systematic review

\section{INTRODUCTION}

In the healthcare setting, an adverse event refers to an injury resulting from medical intervention. ${ }^{[1]}$ Over 33 million individuals are hospitalized annually in the United States (U.S.). ${ }^{[2]}$ Of those hospitalized, one third experience at least one adverse medical event. Among hospitalized individuals that experience an adverse medical event, half will experience more than one event. ${ }^{[1]}$ Each year approximately 210,000-440,000, or 44\%, of hospitalized patients experience one or more preventable medical errors resulting in

\footnotetext{
* Correspondence: MaryAnn Niemeyer, PhD(c), RN-BC; Email: maryann.niemeyer@bjc.org; Address: Christian Hospital, Center for Training \& Development, 11133 Dunn Road, Saint Louis, Missouri 63136, United States.
} 
harm that contributed to their death. ${ }^{[3]}$

Patient safety is the absence of preventable harm during the process of health care. ${ }^{[4]}$ Yet despite deliberate attempts to improve quality of patient care, patient safety is still at the forefront of concerns for healthcare delivery. ${ }^{[5]}$ The Institute of Medicine (IOM) reported on the dire problem of patient safety in healthcare nearly two decades ago, ${ }^{[6]}$ nonetheless patient harm remains a significant concern for hospitals. ${ }^{[3]}$

Nurses are at the forefront of health care and essential for excellence in patient care. ${ }^{[7-9]}$ More than $10 \%$ of the acute care nursing workforce are newly licensed, novice registered nurses (RN) who have a notably higher risk of patient harm during their practice. ${ }^{[10]}$ Novice RNs, those with less than 2 years of practice experience have formal education but limited practical experience. Evidence suggests a relationship between patient safety and number of years of practice. ${ }^{[1-13]}$ Patient mortality is highest among RNs with 2 or fewer years of experience. ${ }^{[14]}$

\section{Background}

Confidence is one of the most influential motivators of behavior a novice RN can possess. Albert Bandura, social cognitive psychologist, believed that self-confidence and performance were inextricably related, and that experiences play a big part in confidence development. ${ }^{[15]}$ Evidence supporting Bandura's research suggests that novice RNs' perception of ability, or self-confidence, is necessary for improving clinical performance. Likewise, the risk of preventable adverse events decreases the closer a novice RN moves towards competent clinical performance. ${ }^{[16,17]}$

Nursing is a practice-based profession with clinical training a critical part of nursing education. Nursing education programs each contain their own set of curricular requirements. RNs are prepared for a wide range of roles and responsibilities caring for patients in a variety of institutional settings (Ballard, 2003). Novice RNs have this formal education but limited practical experience ${ }^{[11-13]}$ often with insufficient exposure to a diverse set of clinical situations. ${ }^{[18-23]}$ Novice RNs often lack confidence in their skills and find the transition from the role of student to working professional $\mathrm{RN}$ particularly challenging. ${ }^{[24]}$

Confidence is a common theme in studies that have examined novice RNs' ability to learn skills required for safe patient care. ${ }^{[25-28]}$ However, few studies focus on novice RN confidence and training strategies in the hospital setting. Investigations of hospital training strategies are needed to build the knowledge base for improving novice $\mathrm{RN}$ confidence for the application of clinical skills used towards safe patient care.
In the hospital setting, novice RNs have increased error rates and severity of error for the first six years of their practice. The risk of error falls by $10.9 \%$ and serious error decreases by $18.5 \%$ with each additional on-the-job year of experience up to six years at which time the risk is diminished to that of experienced RNs with six or more years of hospital practice. ${ }^{[29]}$ Investigators report descriptions of novice RNs as individuals requiring extra advice and guidance for clinical procedure and technical skill deficits. ${ }^{[18,29]}$

Furthermore, the skill mix of novice vs. experienced RNs during patient care can be a concern. ${ }^{[14,30]}$ In the hospital setting, a staffing mix of $50 \%$ novice RNs can significantly degrade quality of care as compared to hospital units that have $20 \%$ novice RNs. ${ }^{[30]}$ Hickey \& Conner (2013) reported a much lower cut-off point for unsafe staffing mix where $20 \%$ novice RN staffing significantly increases the risk of harm to patients. Therefore, in today's fast-paced, complex clinical environment, training strategies in acute care are needed to facilitate the transition of newly graduated, novice RNs into practice to minimize error and patient harm. ${ }^{[11-13]}$

The Joint Commission supports the use of planned, comprehensive training periods for newly graduated, novice RNs so that sufficient knowledge and skill may be acquired to deliver of safe, quality care that meet professional standards of practice. ${ }^{[31]}$ The Robert Wood Johnson Foundation at the Institute of Medicine published recommendations for transforming healthcare that include achieving higher levels of RN continuing education training. ${ }^{[32]}$ Numerous sets of training competencies designed to help bridge the gap from novice to practicing $\mathrm{RN}$ are available from a variety of sources. ${ }^{[33-39]}$ However, continuing education is often fragmented and underdeveloped. ${ }^{[32]}$

To complicate matters, research suggests that novice RNs solve ill-structured problems differently than experienced nurses. The design and support for training novice RNs is therefore more challenging than training experienced RNs. ${ }^{[40]}$ Strategies that are meant to help transition novice RNs into real world practice need to be grounded in evidencebased education where training is integrated with best practice techniques. ${ }^{[41]}$

Although patient safety in healthcare is often hampered by a variety of cultures and organizational changes, efforts to embed patient safety into continuing education must continue. ${ }^{[34,42]}$ The identification of effective evidence-based strategies is central for supporting novice RNs in the profession, for example efforts are underway to replace passive learning experiences with experiential approaches. ${ }^{[43-46]}$ The safety of patients depends on research driven, dedicated patient safety content being integrated into health professional 
curricula and training programs. ${ }^{[34,42]}$

Previous systematic reviews addressing nurse education strategies with a focus on patient safety and confidence have discussed undergraduate RN education, ${ }^{[47]}$ specific training strategies for experienced $\mathrm{RNs}^{[48]}$ and training programs designed for novice RNs such as nurse residency, internships, or orientation programs. ${ }^{[25]}$ The purpose of this ystematic review was to synthesize findings from qualitative, quantitative, and mixed method investigations that examined effective continuing education and training strategies for improving patient safety in hospitals while enhancing confidence among novice RNs.

\section{MeTHODS}

A Boolean search using EBSCOhost search engine was applied for this review. The search was set to show recently published papers within the last ten years, sorted by relevance from January 2007 through August 2017, while searching for the most recent patient-safety focused research-based novice $\mathrm{RN}$ training. The author of this manuscript independently conducted the search and selection process. The articles of the initial search were critically reviewed for relevant data by the title and abstract. These articles were indexed as eligible, potentially eligible, and not eligible. Prospective eligible and potentially eligible full-text reports were reviewed for inclusion through predefined criteria and study quality indicators. Data were extracted, synthesized, summarized, and reported based on the reporting approach, the 'Preferred Reporting Items for Systematic Reviews and Meta-Analyses' (PRISMA). ${ }^{[4]}$ Figure 1 summarizes the study selection process, including identification, screening, eligibility, and inclusion criteria. ${ }^{[50]}$

\subsection{Eligibility criteria}

For this review, post-graduation education development programs refer to education and training programs in a hospital setting, and are synonymous. The terms novice nurse, new nurse, graduate nurse (GN) and newly licensed registered nurse (NLRN) are characterized in the literature as a newly graduated, novice RNs with up to 3 years of experience after graduation. To be included in this review, novice RNs must have experienced some type of continuing education or learning experiences and have hospital based employment. Reports on methods or strategies of education or training provided to undergraduate nursing students or primarily with other allied health learners are excluded. Education must have been delivered, facilitated, or monitored by an experienced educator or clinical team. This review includes peerreviewed, primary literature of original research published in English.

\subsection{Search process}

The following databases were used in this search: CINAHL, Communication \& Mass Media Complete, Education Full Text (H.W. Wilson), Health \& Wellness Resource Center, and Science Direct. Key terms included, ("teach method" OR "education strateg*" or "education method" OR "teach strateg*") AND ("self-efficacy" OR "confidence") AND ("novice nurs*" OR "new graduate nurs*" OR "newly licensed registered nurs*") AND ("patient safety" or "safe patient care").

\subsection{Information sources and study selection}

The initial EBSCOhost search yielded a total of 141 articles. Seven articles were removed as being duplicates. The titles of these remaining 134 potentially relevant publications were screened for eligibility. If eligibility could not be determined by the title, the article was further assessed by reading the abstract. If the eligibility could not be established by the content of the abstract, the entire article was accessed and reviewed for fit. One-hundred and seven records were excluded for not matching the purpose of the study. The remaining full text articles were assessed for eligibility. Fifteen articles were at this point excluded with six studies being undergraduate studies, one with indeterminate documentation of nursing experience, and seven not primary studies. Thus, twelve studies were deemed eligible for inclusion. Seven studies were conducted in the U.S. and five in other countries (see Figure 1). To ensure quality of primary studies with diverse designs within this mixed method systematic review, the evaluation tool "Mixed Methods Appraisal Tool" (MMAT) - Version 2011 was used to assess the eligible qualitative, quantitative, and mixed methods studies for study quality indicators. ${ }^{[51]}$

\section{RESUlTS}

\subsection{Education strategies}

The Results section presents an analysis of education strategies, study design, theoretical framework and key outcomes (confidence, competence).

According to the literature there are multiple strategies available to design education programs for novice RNs focused on patient safety. The main education strategies, in order of high to lower frequency of use, were: (1) simulation-based learning, ${ }^{[26,52-54,56-58,60-62]}$ (2) didactic instruction and preceptored clinical experiences, ${ }^{[26,59]}$ and (3) use of multi-media electronic technologies. ${ }^{[60,61]}$ Case scenarios were reported as adjunct strategies and were used in a majority of studies. ${ }^{[26,52-58,60-62]}$ Student feedback was reported in most of the strategies as being provided through human debriefing experiences, ${ }^{[52-54,56-59,62]}$ through real time computerized feedback, ${ }^{[60]}$ and through real time live human feedback. ${ }^{[26,59]}$ 


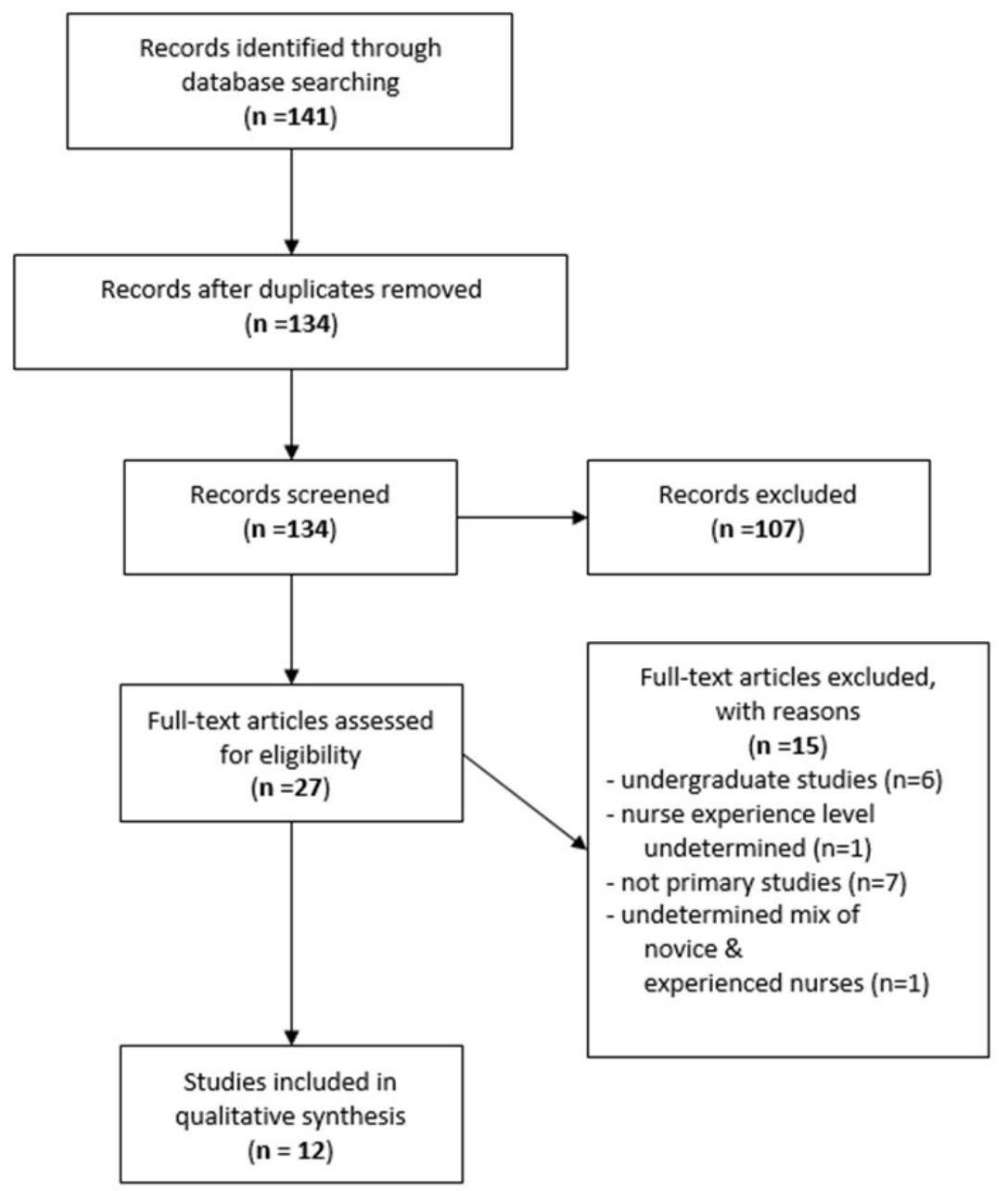

Figure 1. Search process

It is notable that when several strategies were applied together, simulation-based learning was most frequently used as the base strategy. For example, simulation in this review frequently included case study and debriefing. ${ }^{[52-54,56-58,62]}$ In addition, simulation was found to have been combined with other strategies such as lecture, ${ }^{[26,58]}$ skills stations, ${ }^{[26]}$ clinical practice with preceptors ${ }^{[26]}$ and self-directed learning packages. ${ }^{[58]}$

Of the simulation strategies, multiple simulator tools were used. The most common simulator tool was the manikin, ${ }^{[26,52-54,56-58,62]}$ including both high and low fidelity simulator capabilities. The second most frequently used simulator tool was multi-media electronic technologies. ${ }^{[60,61]}$ These can be separated into different types of electronic technologies: a virtual reality task trainer ${ }^{[60]}$ and an audio visual case presentation. ${ }^{[61]}$

High-fidelity simulator manikins are programmed to have a large degree of precision for replicating human clinical reaction. ${ }^{[26,52-54,56,57]}$ Low-fidelity manikins copy or reproduce physical findings but do not interact with the learner. ${ }^{[58,62]}$ Simulations without manikins included multi-media technologies where simulation recreates a partial environment for education training where one or more targeted tasks are performed ${ }^{[60,61]}$ Physical task training tools combined with computer technology replaced real clinical procedure experiences with guided, direct participation. ${ }^{[60,61]}$ Education without any type of simulation included investigation for effectiveness of collaborative interdisciplinary practice experiences, ${ }^{[55]}$ and the effectiveness of classes and clinical experiences. ${ }^{[59]}$ Overall, most studies had small sample sizes.

\subsection{Study design}

Research designs varied within the twelve studies reviewed. Study sample sizes ranged from 10-514 individual novice $\mathrm{RN}$ participants. Of these, three were experimental designs, ${ }^{[57,58,60]}$ including one prospective experimental design, ${ }^{[57]}$ one interventional study, ${ }^{[58]}$ and one experimental pretest posttest, random assignment. ${ }^{[60]}$ There were two mixed methods designs, including one explanatory se- 
quential mixed methods design ${ }^{[55]}$ and one experimental, retrospective design. ${ }^{[62]}$ Four investigations were quasiexperimental, including one prospective study design using pre-test, intervention, and post-test with a non-synchronized, non-equivalent control group ${ }^{[61]}$ two pretest-posttest cohort designs, ${ }^{[53,56]}$ one pretest-posttest single group design, ${ }^{[52]}$ and one longitudinal, non-randomized study. ${ }^{[26]}$ There were two qualitative designs, including one exploratory, semistructured individual interview design, ${ }^{[54]}$ and one individual, unstructured, open-ended interview design. ${ }^{[59]}$

\subsection{Theoretical framework}

Theoretical frameworks and conceptual models serve as foundations for investigations and are used to describe the phenomenon under investigation. Theoretical foundations provide a systematic method for articulating an idea (theory) and the method by which that idea is turned into action (practice). Theory is therefore an important element of the systematic review as it assists the reader with understanding the interpretation of investigational outcomes. In essence, the inclusion of theory in the design of an investigation works as a guide when implementing interventions for clinical practice. The absence of a theoretical framework results in a lack of awareness of the underlying concepts and hinders data extraction and the methodological criteria used to interpret findings. Theory is foundational to scientific study; reports should clearly describe the logic of how the theory operates in the study. ${ }^{[63]}$

About one-half of the studies reported methods guided by a theoretical framework. Beyea et al. (2010), ${ }^{[26]}$ Jung, et al. (2017), ${ }^{[53]}$ Kaddoura (2010), ${ }^{[54]}$ Roche et al. (2013), ${ }^{[57]}$ Spiva et al. (2013), ${ }^{[5]}$ and Tsai et al. (2008) ${ }^{[60]}$ cited no specific theory to describe the underlying concepts in their studies. Pfaff et al. (2014) ${ }^{[55]}$ described the conceptual basis for the interventional strategy as being interprofessional collaboration. Yoo \& Park (2014) ${ }^{[61]}$ reported the intervention to be based on a constructivist framework. Fadale et al. (2014) ${ }^{[52]}$ and Shepherd et al. (2007) ${ }^{[58]}$ reported using Bandura's Self-efficacy theory. Fadale et al. (2014) ${ }^{[52]}$ measured changes in self-efficacy and Shepherd used the theory to describe the impact of learning interventions.

Rhodes et al. (2016) ${ }^{[56]}$ found Dewey's experiential learning a good fit and used this theory as a foundation for examining nurse multidisciplinary simulation. Young \& Burke (2010) ${ }^{[62]}$ used Rogers's theoretical framework to guide the investigation for the exploration of students' selfactualization experiences with simulation. Rhodes, et al. $(2016)^{[56]}$ was the only investigator to have described a theoretical model for debriefing. In this study, Rudolph's advocacy/inquiry provided guidance for creating a psychologi-

Published by Sciedu Press cally safe yet constructive learning environment.

\subsection{Key outcomes}

Outcomes in all studies were investigated by examining the perceptions of novice RNs. Findings were determined using a wide range of data collection and analysis techniques. For the most part, instruments were documented as valid and reliable, with the exception of the SSCS tool ${ }^{[26]}$ and the ACES validation form ${ }^{[62]}$ where the design of the instruments was described but presented without clear documentation of validation or reliability. In some studies, confidence was measured and discussed in terms of self-efficacy. Others discussed the improved confidence of novice RNs for conducting specific clinically-important skills. Competence improved in novice RNs across all studies.

\subsubsection{Confidence}

Thematic analysis of the data resulted in finding conceptual patterns among the sources. Although training strategies varied, confidence was found to be a strong theme in ten of the twelve studies. Notably, the investigation reported by Pfaff et al. (2014) ${ }^{[55]}$ on interprofessional collaboration had particularly interesting findings that suggest certain services may help facilitate novice RN confidence development. Here, acute care RNs experiencing interprofessional collaboration developed higher confidence levels compared to those in community care and long term care employment.

Other factors related to enhanced confidence were found to be the novice RNs' proximity to the educator, accessibility to the educator, proximity to manager, accessibility of manager, number of team strategies, number of different disciplines worked with daily, and satisfaction with the team. ${ }^{[55]}$ Interview data corroborated the participants' reported increases of confidence in supportive relationships, respect, knowledge and interprofessional collaborative experience. ${ }^{[55]}$ Confidence in the novice RNs' ability to think critically in terms of priority setting, decision making, communication, and reporting improved during simulated experiences. ${ }^{[53]}$ Elsewhere, confidence levels improved after high priority education was delivered in classes and though clinical rotations. Confidence also improved over time and within different themes of learning such as having experience, learning to manage time, and learning to communicate. ${ }^{[59]}$

Several simulation studies also examined confidence. ${ }^{[26,53,56,58-60]}$ Novice RN confidence improved over time in simulation experiences ${ }^{[26,59]}$ and in the clinical environment up to 18 months after the simulation training. ${ }^{[56]}$ Analysis demonstrated positive feedback in confidence with mastering skills, ${ }^{[60]}$ and gains in knowledge were also associated with the improvement of confidence. ${ }^{[58]}$ Others found 
simulation experiences to boost confidence for using staffing resources. ${ }^{[62]}$

Confidence improved with practice in solving clinical problems. In case-based learning (CBL), confidence levels improved for novice RNs actively engaged in problem-solving during the viewing of video re-enactment simulated casebased scenarios. ${ }^{[61]}$ This study included a non-equivalent control group. The education was delivered by two different specialty groups of professionals during two different periods of time. The traditional lecture was delivered by the Quality Management Department to novice RNs in 2009 whereas the professional video case reenactment was created and delivered in 2010 by the investigators and a case-based learning education consultant. ${ }^{[61]}$

\subsubsection{Competence}

Confidence was a second thematic pattern found within the data. Of the twelve studies included in this review, all twelve found an improvement in competence levels among novice RNs. ${ }^{[26,52-62]}$ Competency in novice RNs was described as the ability to meet entry-level expectations of the nursing profession. ${ }^{[26]}$ More frequently, however, competency was defined in terms of specific clinical skill or skills, such as in communication, ${ }^{[53,55,57,59,62]}$ assessment skills ${ }^{[57-59]}$ critical thinking skills, ${ }^{[53,54,61,62]}$ prioritization skills, ${ }^{[62]}$ advanced nursing skills, ${ }^{[52,60]}$ modest, steady increases in knowledge, ${ }^{[56]}$ and in overall clinical performance. ${ }^{[26,54]}$

As noted, competence was measured in multiple skill types. For example, Pfaff et al. (2014) found that communication skills improved for participants who engaged in interprofessional educational opportunities, with qualitative data supporting the quantitative findings for improved communication skills. ${ }^{[55]}$ Communication also improved during novice RNs orientation experiences. Professional growth was found to improve with time as novice RNs enhance their communication skills. ${ }^{[59]}$ While exploring novice $\mathrm{RN}$ experiences of simulation, research findings suggest that both clinical and simulation experiences improve novice $\mathrm{RN}$ communication while fostering critical thinking skills, ${ }^{[62]}$ and increasing knowledge. ${ }^{[56]}$ Interestingly, communication performance data demonstrated no statistical significance between the simulation intervention group and the written case studies control group ${ }^{[57]}$ however, the intervention group (simulation) performed better on safety behaviors than the control group (written case studies). This suggests that practicing scenarios with hands-on experiences is more effective than discussion of the scenarios without hands-on practice.

In an investigation by Shepherd et al. (2007), ${ }^{[58]}$ three learning learning interventions were analyzed for clinical reactions of novice RNs: a self-directed learning package, a self- directed learning package with two scenario-based didactic lectures using PowerPoint workshops, and a self-directed learning package with two low-fidelity manikin simulation sessions. Novice RNs' patient assessment skills improved significantly during simulation interventions as compared to control groups using scenario-based didactic lecture with PowerPoint. Novice RNs' patient assessment skills also improved significantly during simulation interventions as compared to the control groups using the self-directed learning package. ${ }^{[58]}$

Critical thinking as a competency was also measured. Beyea (2010) found critical thinking proficiency to improve during manikin simulations as novice RNs learned to "think on the fly". [26] Young \& Burke (2010) also found critical thinking to be enhanced using simulation during the Advanced Clinical Education and Simulation (ACES) course. In a mixed method study, novice RNs were surveyed on their course experiences. Novice RN participants reported that the manikin simulation course fostered their critical thinking competency during their transition into skilled and safe practicing RNs. ${ }^{[62]}$

Other investigations reporting improved competency discussed enhanced prioritization skills, ${ }^{[62]}$ advanced nursing skills, ${ }^{[52,60]}$ and overall clinical performance. ${ }^{[26,54]}$ Although novice RNs perceived the simulation portion of the Advanced Clinical Education and Simulation (ACES) course to have improved their prioritization skills, a larger majority thought that the post-simulation debriefing sessions were more valuable for learning to prioritize. In an investigation by Fadale et al. (2014), advanced nursing skills were found to improve during port-a-cath simulations using electronic multi-media. Self-directed learning during virtual reality simulations was found to be an effective process for improving advanced skills with knowledge gains and improved clinical procedure skills. ${ }^{[60]}$ Simulation also proved to be helpful for learning vasopressor titration skills, an advanced nursing skill. ${ }^{[52]}$ These are especially useful findings as they may aid in improving patient safety training as simulation experiences were found to effectively prepare novice RNs to care for very sick patients. ${ }^{[26,54]}$

\section{Discussion EDUCATION STRATEGIES}

Figure 2 provides a representation of selected studies' education strategy with findings for confidence and competence. Improvements to novice RNs' confidence were found in seven out of eight investigations that included manikin simulation. The Discussion section herein presents key evidence from each of the education strategies, and discusses how confidence and competence play a role in each of the findings. 


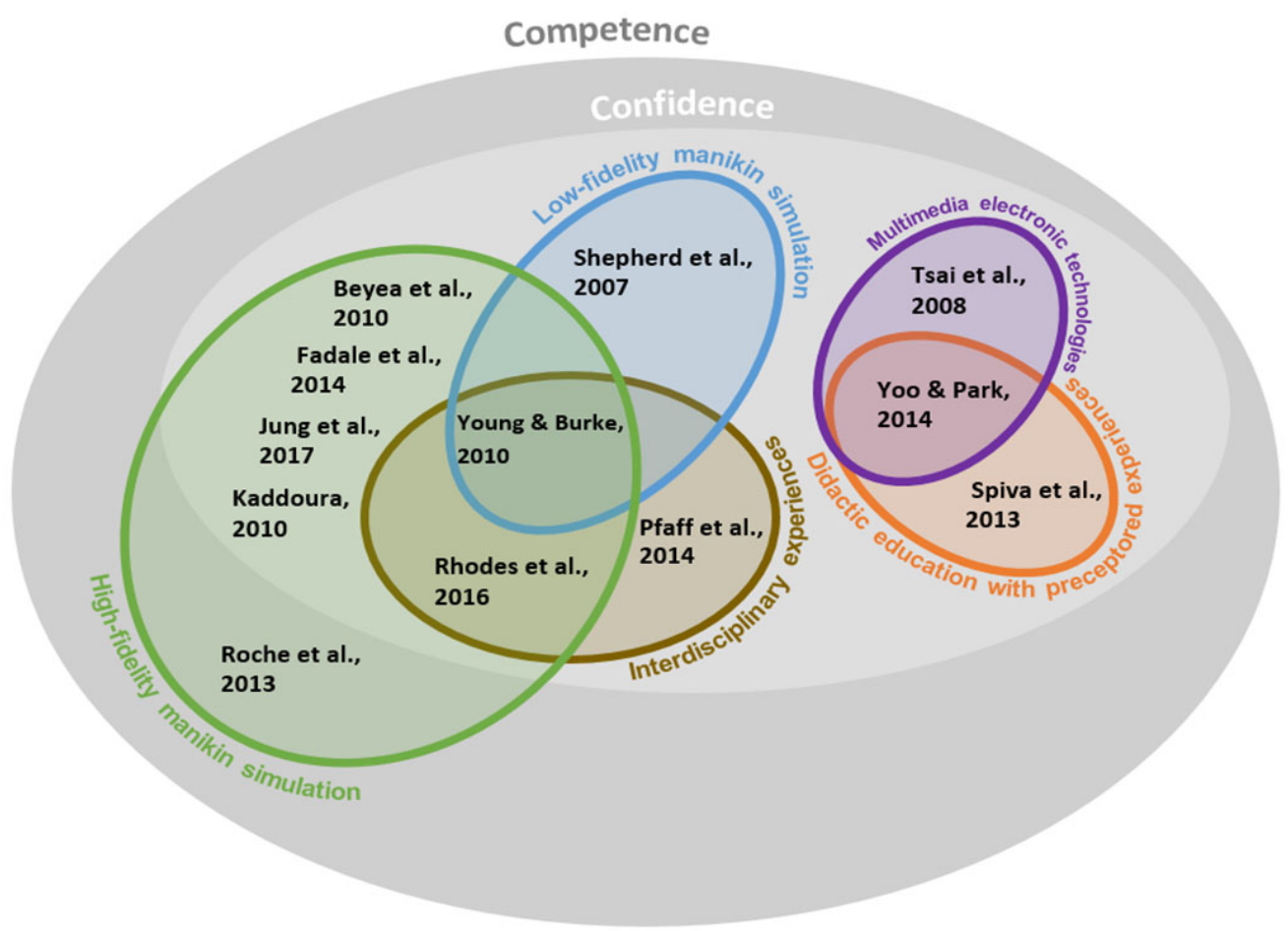

Figure 2. Illustration of commonalities and distinguishing features among education strategies reported to have improved confidence and competence in novice RNs

Novice RNs require specialized teaching strategies as they are at increased risk of medical error resulting in patient harm. Effective patient safety education for novice RNs working in hospitals is an understudied topic. The implications for the findings in this review could affect the direction of nurse training investments. Simulation in healthcare seems to be the overall favorite as an interactive technique replacing real experiences with guided experiences. ${ }^{[64]}$ Depicting substantial aspects of patient care, simulated nursing experiences are one option among numerous strategies available to supplement education and training required to increase novice RN confidence and competence needed for safe patient care. ${ }^{[26,52-54,57,58,62]}$ Simulation has been widely accepted and used in academia for the clinical education of undergraduate nursing students. ${ }^{[47,65]}$ Few studies, however, have focused on novice RN hospital-based simulation training for continuing education. ${ }^{[53,56,62,66]}$ Previous literature indicates that simulation could increase safety awareness by widening the scope of simulated experiences to include potential errors and strategies for resolution. ${ }^{[57,67]}$ Moreover, a recent meta-analysis affirms the findings in this systematic review for simulation having a positive effect as a continuing education strategy with RNs, improving both knowledge and performance outcomes. ${ }^{[68]}$

On a basic level, simulation is the interactive technique for enhancing the reaction of participants to a high risk skill. ${ }^{[64]}$
It can be a substitution for the real thing, such as using a manikin or a human being for simulating a healthcare scenario. Although simulation does not require a manikin, training may be enhanced by its use. Simulation in any form must match learners' needs. ${ }^{[69]}$ Scenarios using simulation methods can range from focused clinical education to mass trauma scenarios. Simulation, however, should be seen as an exercise not necessarily dependent how well the simulation matches the realism of the clinical situation. Instead, the objective should be for the trainee and the trainer to skillfully utilize simulation to gain knowledge and experience. ${ }^{[69]}$ Simulated scenarios can be designed to be cost-effective strategies for providing continuing education. ${ }^{[69]}$

\subsection{Low and high-fidelity manikin simulation}

A key advantage to low fidelity simulation is that it is costeffective and portable. Low fidelity simulators can be mobilized to facilitate learning in a contextualized, real-world setting. Accessibility to training could improve training compliance and reduce time spent away from clinical care. Scenarios, when repeated frequently, may serve to reinforce training by decreasing the deterioration of learned concepts. Moreover, regularly scheduled simulations could help with the comfort level of managing critical patient events, improving novice $\mathrm{RN}$ ability for early recognition of patient deterioration and crisis situation interventions with increased 
frequency of training events. A disadvantage is that that low fidelity simulators do not respond to the actions of the novice $\mathrm{RN}$ because there is no life-like feedback. Thus, novice RNs learning in low fidelity situations must be given information verbally about the scenario with their patient during their care. $^{[58,62]}$

High fidelity simulation, on the other hand, allows for the realism of more complex patient care scenarios. High fidelity simulators provide immediate, life-like feedback to the learner. Confidence and competence were each found to be important results in simulation strategies. These improvements are likely due to the gains in experience novice RNs receive by role-playing and practicing critical clinical skills required for safe patient care. Through the fidelity of the simulation, just-in-time feedback assists with supporting health care concepts learned in the classroom. Moreover, high fidelity simulations seem to help with improving novice RN ability for early recognition of patient deterioration and management of crisis situation interventions to prevent failure to rescue situations.

However, high-fidelity simulation has its drawbacks. High fidelity simulators are expensive and less mobile than low fidelity simulators. Because of the realistic, life-like responses, high fidelity simulation can be intimidating for the novice $\mathrm{RN}$ learner. In addition, high fidelity simulators require more extensive educator training. Even with experienced nurse trainers, the complexity of a high fidelity simulator can be daunting as educators learn to use the computerized programing of the simulator. Feedback is essential to novice RN learning, and is best provided by an experienced and well trained simulation educator. ${ }^{[26,52-54,56,57]}$

\subsection{Multi-media electronic technologies}

Virtual reality and audio-visual simulations provide costeffective user education that can be practiced over and over again without the need for an onsite trainer. This method can employ case scenarios for training purposes, or can demonstrate proper technique of a specific clinical skill. The downside is that the education must be created in advance using technology that may be challenging to learn, or costprohibitive as an initial purchase. Another limitation is that there is no learner feedback unless a trainer is onsite to interact with the students. ${ }^{[60,61]}$ Simulation without learner feedback is likely a significant limitation, as necessary information required for effective learning is absent, potentially affecting learner outcomes.

\subsection{Interdisciplinary experiences}

Interdisciplinary experiences provide well rounded learning in the clinical setting. Connecting novice RNs with formal leaders and members of the interdisciplinary team can increase novice $\mathrm{RN}$ confidence in team communication. A limitation is that the experiences, when practiced on live hospitalized persons, produced safety risks. One way to minimize risk is to simulate interdisciplinary scenarios; however, simulated interdisciplinary scenarios require considerable planning and could remove some clinicians away from patient care. ${ }^{[55]}$

\subsection{Didactic learning with preceptored experiences}

Preceptored experiences also served as non-traditional methods for training novice RNs. ${ }^{[59]}$ Preceptored education strategies have similar outcomes to previous findings that "master apprenticeships" are valuable for the clinical training of novice RNs. ${ }^{[69]}$ However, there are too few preceptors in the workplace, placing undue stress on the few that remain, further exacerbating risks to patient safety. Moreover, novice RNs can sometimes cause patient harm on patients by practicing on patients before they are safe care clinicians. Findings from this literature review, on the other hand, note that the use of simulated clinical experiences ensure that those initial novice RN high risk or safety focused experiences can be instead practiced in a way that cannot harm real patients.

For example, coupled with preceptored clinical experiences and individual training sessions, the effectiveness of scheduled classes for novice $\mathrm{RN}$ education can enhance overall clinical confidence and skill competence. ${ }^{[59]}$ Interestingly, there is no added value with the addition of self-directed learning packets with didactic instruction for the improvement of competence and confidence while combined with simulated learning experiences. ${ }^{[58]}$ Didactic learning is an efficient method for quickly dispersing small bits of information in short bursts of time such as for providing content in orientation. Preceptored experiences are an excellent resource for novice RNs, providing some protection from harm for novice RNs and the patient during the novice RNs' first weeks and months of patient care. Individual training is helpful for skills such as IV and port-a-cath insertions, pump programming, Foley catheterizations, or chest tube maintenance, where during school little to no practice was provided.

\subsection{Implications for practice}

Americans are older, sicker, and more expensive to care for than at any time in history. Almost 20 years after the Institute of Medicine reported on hospital safety, "To Err is Human: Building a Safer Health System", medical error is the third leading cause of death behind breast cancer, AIDS, an motor vehicle crashes. ${ }^{[70]}$ Previous efforts to improved safety in hospitals has stalled. ${ }^{[71]}$ In hospitals, novice RNs have the greatest risk for medical error and severity of error. Novice 
RNs learn differently than experienced RNs because they do not have experience to draw from. This makes their education that much more challenging. What remains unclear is how to best educate patient safety to the novice $\mathrm{RN}$ to minimize or prevent medical error.

Simulation strategies that work well for the transition from novice to practicing $\mathrm{RN}$ are an important goal for patient safety initiatives in novice RN education. Simulation has proven a positive alternative to traditional continuing education in novice RNs. The most successful combinations of education patterns, those that have not yet been recognized as proven strategies, might be uncovered as important features for professional development in the novice $\mathrm{RN}$ transition to competent RN. Moreover, novice RNs experiencing positive transitions to safe, practicing RN create happier novice RNs, potentially raising retention rates for this group. ${ }^{[72]}$ In addition, overall improved quality of hospital care lowers the risk of medical error and has the potential for producing happier patients as evidenced with improved patient satisfaction scores. ${ }^{[73]}$

Today's healthcare setting care is complex. Caring for patients safely is more challenging than ever before. In this review, multiple examples of learning strategies have helped describe effective training for post-graduate novice RNs, including manikin simulation, multi-media electronic technologies, interdisciplinary experiences, and didactic learning with preceptored experiences. Simulation as a non-traditional learning approach included low and high fidelity manikins, and multi-media electronic technology simulation. It appears that simulation is the most adaptable of the three dominant strategies reviewed. Simulation can be used independently or combined with other strategies; in addition, simulation seems to provide tremendous location flexibility and cost efficiency, and can be molded around objectives designed to fit the need of the novice RN.

In the hospital, both high and low fidelity simulation can help with improving novice $\mathrm{RN}$ ability for early recognition of patient deterioration and management of crisis situation interventions, and each strategy has its drawbacks. While high fidelity experiences provide immediate, life-like feedback to the learner, they are expensive and can require a laboratory setting with highly trained clinical educators. Low-fidelity simulation can be used to train novice RNs in a variety of settings for multiple patient safety scenarios. Simulation sessions where the novice RNs needs to have hands-on practical exercise is possible with both with lab and in-situ novice RN training exercises. For example, when a patient is transferred to a different level of care, multiple patient safety assessments need to be completed. Scenarios that include safety

Published by Sciedu Press checks such as physical assessment, medication verification, IV assessment, IV pump and other equipment assessments can be completed by low-fidelity manikin simulation in either setting, lab or in-situ. Especially in the clinical setting, simulations are an effective strategy in preparing novice RNs for the unexpected clinical scenario. ${ }^{[58]}$

Simulation was found to be the overwhelming predictor for novice RN gains in confidence and practice competence. Based on the articles analyzed for this systematic review, it seems clear that simulation is an effective choice for training novice RNs as they transition into confident, competent RNs who practice safely. Nurse educators with experience in acute care and simulation training techniques can add to novice RNs' patient care experiences by using simulation in the acute care setting. Simulation strategies in healthcare are a useful tool for addressing error and improving teamwork and communication. Novice RNs are able to improve their confidence and competency when provided simulated training, and view simulated scenarios as training experiences capable of producing a change in their behavior for the acquisition of new skills.

The use of effective teaching strategies has been found to be especially important for studying complex concepts such as patient safety in clinical education. Simulation for training novice RNs was demonstrated as a powerful and effective strategy, and each of the nine investigations that used simulation in some form experienced an improvement in competence, confidence, or both during novice RN training. Simulation has shown to be beneficial to novice RNs learning to care for patient safely, and simulation research with novice RNs is one unique course of action that might help prevent future medical error tragedies among novice RNs.

\section{Recommendations for further research}

Diverse forms of data sources and analysis methods are important in research design because multiple, varied data sources and perspectives help strengthen the validity and credibility of the systematic analysis. Therefore, suggestions for further research include a more comprehensive synthesis of data in the form of a mixed method systematic review using a team approach. Moreover, increasingly complex hospital environments, greater numbers of patients with multiple, chronic health care problems, limited preparation time, and scarce numbers of clinical sites result in novice RNs receiving limited hands-on opportunities-the very experiences required to prepare them to function as confident, competent, safe RNs in today's hospital setting. The findings from this review support Benner's understanding of novice RN professional development from novice to expert nurse. ${ }^{\text {[7] }}$ Nurses may be better able to conceptualize and therefore 
identify appropriate courses of actions through repeated practice, including simulated experiences. Thus, education that is designed fill gaps in the preparation and readiness of novice RNs will most likely be fulfilled through the development of simulation interventions to be used in the hospital setting. Future research should additionally focus on the prevention of "failure to rescue" events by novice RNs. By gaining confidence and competence particularly in caring for lower volume emergent situations such as a patient experiencing clinical deterioration, novice RNs will greatly reduce their risk of medical error and hospitals will achieve improved safety for their patient population.

\subsection{Strengths and limitations}

This review examines strategies for teaching novice RNs learning to care for patients safely. A strength of the review is the structured search of available literature within peerreviewed primary studies for strategies used to train novice RNs. The review outlines strategies for supporting novice RNs, and describes the less successful strategies. Educators are able to take these results and use them for improving the development of unique, effective post-graduate novice RN training. The limitation of the ten-year search range and by limiting the sample to novice RNs in the hospital setting many have reduced evidence found on patient safety continuing education strategies. Also, the data field search strategy using EBSCOhost resulted in capturing a limited number of databases. In the future a broader set of keywords might help increase the return on the number of databases, therefore increasing the number and variety of potentially eligible articles. In addition, the inclusion of a second search engine for exploring biomedical literature such as PubMed, ${ }^{[75]}$ and searching databases individually, could yield an increase in search results. ${ }^{[76]}$

Another limitation is that approximately half of the studies were completed outside of the United States increasing the divergence of clinical practice and settings. This variability of locations may have contributed to potential differences in learner perspectives. Across studies, education strategy was also diverse and varied widely both in the base and adjunct education strategies thereby limiting generalizability of the results. In addition, the assessment of novice RNs clinical ability differed and in some cases was reported as challenging. A higher quality assessment of novice RN clinical ability could help support methods for evaluating novice RN learning achievements and serve as a starting point for a greater focus on post-graduate education development. Moreover, outcomes for each strategy were measured using different instruments and direct observation was described as not always accurate due to the techniques used in assessment and potential inter-rater differences. Novice RNs rating their outcome perceptions each have their own human history of clinical and life experiences and so perceptions when rating learning experiences are likely to vary as well. When data collection methods vary, outcomes comparisons across studies are challenging.

To reduce publication selection bias this systematic review encompasses qualitative, quantitative, and mixed methods research. A qualitative synthesis, this report does not include meta-analysis. The omission of a meta-analysis might be considered a limitation by some readers. However, the outcomes of the selected studies were derived by both quantitative and qualitative data. Qualitative methods are based on observation and do not use figures and numbers. In addition, the strategies reviewed covered multiple educational modalities. Thus, a mathematic summary meta-analysis of the outcomes using the available statistical results would provide little insight for readers. ${ }^{[77]}$

\section{Conclusion}

Continued evidence collection and the assessment surrounding safe patient care education facilitates a better understanding of how novice RNs can improve their practice confidence and effectively learn to keep patients safe. This literature review investigated strategies used to train novice RNs for safe patient care and spotlights the paucity of evidence focused on confidence and safety in hospital-based continuing education. A highlight of this review reveals several interesting findings. Simulation, far outnumbering other reported strategies, seems to be gaining acceptance as an effective option that can be used to increase novice $\mathrm{RN}$ confidence for the application of clinical skills used to care for patients safely. The pathway to get there, according to the literature, is to ensure simulated experiences include potential errors and strategies for resolving those errors. Combined with an increased focus on patient safety, hands-on simulated clinical experiences appear to positively affect novice RNs' abilities to obtain relevant clinical experience and, by making connections through repeated practice, develop higher levels of thinking. Serving as a guide for future research in the exploration for novel methods of delivering simulation training, this review supports the call by the Institute of Medicine, ${ }^{[32]}$ the Agency for Healthcare Research and Quality, ${ }^{[78]}$ and the National League for Nurses ${ }^{[39]}$ to develop, test, and evaluate strategies with a focus on simulation for improving the safe delivery of health care. The overarching goal is to create a foundational knowledge base from which to draw from in planning the investigation of simulation for behavioral changes of novice $\mathrm{RN}$ at the patient bedside, and later for the impact of learning on patient outcomes. 


\section{ACKNOWLEDGEMENTS}

No funds were obtained for completion of this review. The author received assistance with manuscript construction through the support of course instructor Dr. Maithe Enriquez; and with manuscript editing through the support of dissertation advisor Dr. Amy Vogelsmeier at Sinclair School of Nursing, University of Missouri Columbia, Columbia, Missouri, and Dorothy Votoupal-Hayes, retired language teacher, Marquette Catholic High School, Alton, Illinois. Eric Niemeyer, computer engineering student at Southern Illinois University Edwardsville, assisted with illustration design.

\section{REFERENCES}

[1] Classen DC, Resar R, Griffin F, et al. "Global trigger tool" shows that adverse events in hospitals may be ten times greater than previously measured. Health Affairs. 2011; 30: 581-9. https: //doi.org/10.1377/hlthaff.2011.0190

[2] AHA. Trendwatch chartbook. A-32. 2015.

[3] James JT. A new, evidence-based estimate of patient harms associated with hospital care. Journal of Patient Safety. 2013; 9: 122-8. https://doi.org/10.1097/PTS.0b013e3182948a69

[4] Donaldson SL. Patient safety 2014. Available from; http://www . who.int/patientsafety/about/en/

[5] Chassin MR, Loeb JM. High-reliability health care: Getting there from here. Milbank Quarterly. 2013; 91: 459-90. https://doi.or $\mathrm{g} / 10.1111 / 1468-0009.12023$

[6] Kohn LT, Corrigan JM, Donaldson M. To err is human: Building a safer health system. vol. 627. National Academies Press; 2000.

[7] Aiken LH, Clarke SP, Sloane DM, et al. Hospital nurse staffing and patient mortality, nurse burnout, and job dissatisfaction. American Medical Association. 2002; 288: 1987-93. https://doi.org/10 $.1001 /$ jama. 288.16.1987

[8] Lacey SR, Cox KS. Nursing: Key to quality improvement. Pediatric Clinics of North America. 2009; 56: 975-85. https://doi.org/ $10.1016 / j \cdot p c 1.2009 .05 .004$

[9] Sales A, Sharp N, Li YF, et al. The association between nursing factors and patient mortality in the Veterans Health Administration: The view from the nursing unit level. Medical Care. 2008; 46: 938-45. PMid:18725848 https://doi.org/10.1097/MLR.0b013e3181 $791 \mathrm{a} 0 \mathrm{a}$

[10] Berkow S, Virkstis K, Stewart J, et al. Assessing new graduate nurse performance. Nurse Educator. 2009; 34: 17-22. https://doi .or g/10.1097/01. NNA.0000339477.50219.06

[11] Jewell A. Supporting the novice nurse to fly: A literature review. Nurse Education in Practice. 2013. https://doi.org/10.1016/ j.nepr.2013.04.006

[12] Slaikeu KN. Addressing the preparation/practice Gap: A new era, new approach. Nurse Leader. 2011; 9: 46-9. https://doi .org/10 $.1016 / \mathrm{j} . \mathrm{mnl} .2011 .01 .005$

[13] Morrow S. New graduate transitions: Leaving the nest, joining the flight. Journal of Nursing Management. 2009; 17: 278-87. https://doi.org/10.1111/j.1365-2834.2008.00886.x

[14] Hickey PGKCM, Connor J. The effect of critical care nursing and organizational characteristics on pediatric cardiac surgery mortality in the United States. Journal of Nursing Administration. 2013; 43: 637-44. https://doi.org/10.1097/NNA. 0000000000000005

[15] Bandura A. Self-efficacy: The exercise of control. New York: W.H. Freeman and Company; 1997.

[16] Ulrich B, Krozek C, Early S, et al. Improving retention, confidence, and competence of new graduate nurses: Results from a 10-year longitudinal database. Nursing Economics. 2010; 28: 363-75. PMid:21291057

Published by Sciedu Press
[17] Aronson B, Glynn B, Squires T. Competency assessment in simulated response to rescue events. Clinical Simulation in Nursing. 2012; 8: e289-e295. https://doi.org/10.1016/j.ecns.2010.11.006

[18] Hickey MT. Preceptor perceptions of new graduate nurse readiness for practice. Journal for Nurses in Professional Development. 2009; 25: 35-41. https://doi.org/10.1097/NND.0b013e318194b5 $\mathrm{bb}$

[19] Saintsing D, Gibson LM, Pennington AW. The novice nurse and clinical decision-making: How to avoid errors. Journal of Nursing Management. 2011; 19: 354-9. https://doi.org/10.1111/j. 1365-2834.2011.01248. $\mathrm{x}$

[20] Benner P, Hughes RG, Sutphen M. Clinical reasoning, decisionmaking, and action: Thinking critically and clinically. Patient Safety and Quality: An Evidence-Based Handbook for Nurses. 2008; 87-109 p.

[21] Jamshidi N, Molazem Z, Sharif F, et al. The challenges of nursing students in the clinical learning environment: A qualitative study. The Scientific World Journal. 2016. https://doi.org/10.1155/ 2016/1846178

[22] Heidari MR, Norouzadeh R. Nursing students' perspectives on clinical education. Journal of Advances in Medical Education \& Professionalism. 2015; 3: 39. PMid:25587554

[23] Valiee S, Moridi G, Khaledi S, et al. Nursing students' perspectives on clinical instructors' effective teaching strategies: A descriptive study. Nurse Education in Practice. 2016; 16: 258-62. PMid:26489603 https://doi.org/10.1016/j.nepr.2015.09.009

[24] Sparacino LL. Faculty's Role in Assisting New Graduate Nurses' Adjustment to Practice. International Journal of Nursing. 2016; 2: 1-9. https://doi.org/10.1177/2377960816635182

[25] Edwards D, Hawker C, Carrier J, et al. A systematic review of the effectiveness of strategies and interventions to improve the transition from student to newly qualified nurse. International Journal of Nursing Studies. 2015; 52: 1254-68. https://doi.org/10.101 6/j.ijnurstu. 2015.03.007

[26] Beyea SC, Slattery MJ, von Reyn LJ. Outcomes of a simulation-based nurse residency program. Clinical Simulation in Nursing. 2010; 6: e169-e175. https://doi.org/10.1016/j.ecns.2010.01.005

[27] Askew T, Trotter TL, Vacchiano S, et al. Avoiding failure to rescue situations: A simulation exercise for oncology nurses. Clinical Journal of Oncology Nursing. 2012; 16: 530. https ://doi .org/10.1 188/12.CJON. 530-532

[28] Massey D, Chaboyer W, Anderson V. What factors influence ward nurses' recognition of and response to patient deterioration? An integrative review of the literature. Nursing Open. 2017; 4: 6-23. https://doi.org/10.1002/nop2.53

[29] Westbrook JI, Rob MI, Woods A, et al. Errors in the administration of intravenous medications in hospital and the role of correct procedures and nurse experience. BMJ Quality \& Safety. 2011; 20: 1027-34. https://doi.org/10.1136/bmjqs-2011-000089

[30] Kanai-Pak M, Aiken LH, Sloane DM, et al. Poor work environments and nurse inexperience are associated with burnout, job dissatisfac- 
tion and quality deficits in Japanese hospitals. Journal of Clinical Nursing. 2008; 17: 3324-9. https://doi.org/10.1111/j.1365 $-2702.2008 .02639 . x$

[31] TJC. Health care at the crossroads: Strategies for creating and sustaining community-wide emergency preparedness systems. Joint Commission on Accreditation of Healthcare Organizations; 2003.

[32] IOM. The future of nursing: Leading change, advancing health Washington, DC: The National Academies Press; 2011.

[33] McGuinn K. Graduate-Level QSEN competencies knowledge, skills and attitudes. Washington: American Association of Colleges of Nursing; 2012.

[34] Ginsburg L, Castel E, Tregunno D, et al. The H-PEPSS: An instrument to measure health professionals' perceptions of patient safety competence at entry into practice. BMJ Quality \& Safety. 2012; 676-84 p. https://doi .org/10.1136/bmjqs-2011-000601

[35] Greiner AC, Knebel E. Health professions education: A bridge to quality. 500 Fifth Street, N.W. Washington, DC 20001: National Academies Press; 2003.

[36] Emanuel L, Combes J, Hatlie M. The patient safety education project. Participant's Handbook Chicago: PSEP. 2008.

[37] Boeing H, Oberritter H, Daniel H. 12th European Nutrition Conference (FENS). Ann Nutr Metab. 2015; 67: 1-20.

[38] Walton M. Patient safety curriculum guide: Multi-professional edition: Global evaluation study. Malta: World Health Organisation. 2011.

[39] NLN. Continuing education program mission and goals. 2600 Virginia Avenue, NW. The Watergate, 8th Floor Washington, DC 20037: National League for Nursing; 2014.

[40] Sarsfield E. Differences between novices' and experts' solving illstructured problems. Public Health Nursing. 2014; 31: 444-53. https://doi.org/10.1111/phn.12100

[41] Robinson BK, Dearmon V. Evidence-based nursing education: Effective use of instructional design and simulated learning environments to enhance knowledge transfer in undergraduate nursing students. Journal of Professional Nursing. 2013; 29: 203-9. https://doi.org/10.1016/j.profnurs. 2012.04.022

[42] Catchpole K. Spreading human factors expertise in healthcare: Untangling the knots in people and systems. BMJ Qual Saf. 2013; 22: 793-7. https://doi.org/10.1136/bmjqs-2013-002036

[43] Coram C. Expert role modeling effect on novice nursing students' clinical judgment. Clinical Simulation in Nursing. 2016; 12: 385-91. https://doi.org/10.1016/j.ecns.2016.04.009

[44] Schams KA. The impact of high fidelity simulation on situational awareness of cardiac related obstetric emergencies in novice nurses. Doctor of Nursing Practice Systems Change Projects. 2013.

[45] Kyrkjebø JM, Bratteb $\varnothing$ G, Smith-Strøm H. Improving patient safety by using interprofessional simulation training in health professional education. J Interprof Care. 2006; 20: 507-16. https ://doi .org/ $10.1080 / 13561820600918200$

[46] Walshe N, O'Brien S, Murphy S, et al. Integrative learning through simulation and problem-based learning. Clinical Simulation in Nursing. 2011. https: //doi.org/10.1016/j.ecns. 2011.08.006

[47] Cant RP, Cooper SJ. Use of simulation-based learning in undergraduate nurse education: An umbrella systematic review. Nurse Education Today. 2016. http://doi.org/10.1016/j.nedt.2016.11.015 0260-6917

[48] Meurling L, Hedman L, Sandahl C, et al. Systematic simulationbased team training in a Swedish intensive care unit: a diverse response among critical care professions. BMJ Quality \& Safety 2013: bmjqs-2012. https://doi.org/10.1136/bmjqs-2012-0 00994
[49] Moher D, Liberati A, Tetzlaff J, et al. Preferred reporting items for systematic reviews and meta-analyses: The PRISMA statement. Annals of Internal Medicine. 2009; 151: 264-9.

[50] Liberati A, Altman DG, Tetzlaff J, et al. The PRISMA statement for reporting systematic reviews and meta-analyses of studies that evaluate health care interventions: explanation and elaboration. PLoS Medicine. 2009; 6: e1000100. https://doi.org/10.1136/bmj . b2700

[51] Pluye P, Robert E, Cargo M, et al. Mixed methods appraisal tool (MMAT) version 2011. Proposal: A mixed methods appraisal tool for systematic mixed studies reviews, McGill University, Department of Family Medicine; 2011.

[52] Fadale KL, Tucker D, Dungan J, et al. Improving Nurses' Vasopressor Titration Skills and Self-Efficacy via Simulation-Based Learning. Clinical Simulation in Nursing. 2014; 10: e291-e299. https://doi.org/10.1016/j.ecns.2014.02.002

[53] Jung D, Lee SH, Kang SJ, et al. Development and evaluation of a clinical simulation for new graduate nurses: A multi-site pilot study Nurse Education Today. 2017; 49: 84-9. https ://doi .org/10.1 016/j.nedt. 2016.11.010

[54] Kaddoura MA. New graduate nurses' perceptions of the effects of clinical simulation on their critical thinking, learning, and confidence. The Journal of Continuing Education in Nursing. 2010; 41: 506-16. https://doi .org/10.3928/00220124-20100701-02

[55] Pfaff KA, Baxter PE, Jack SM, et al. Exploring new graduate nurse confidence in interprofessional collaboration: A mixed methods study. International Journal of Nursing Studies. 2014; 51: 1142-52. https://doi.org/10.1016/j.ijnurstu.2014.01.001

[56] Rhodes CA, Grimm D, Kerber K, et al. Evaluation of nurse-specific and multidisciplinary simulation for nurse residency programs. Clinical Simulation in Nursing. 2016; 12: 243-50. https://doi.org/ 10.1016/j. ecns. 2016.02.010

[57] Roche J, Schoen D, Kruzel A. Human patient simulation versus written case studies for new graduate nurses in nursing orientation: A pilot study. Clinical Simulation in Nursing. 2013; 9: e199-e205. https://doi.org/10.1016/j.ecns.2012.01.004

[58] Shepherd IA, Kelly CM, Skene FM, et al. Enhancing graduate nurses' health assessment knowledge and skills using low-fidelity adult human simulation. Simulation in Healthcare. 2007; 2: 16-24. https://doi.org/10.1097/SIH.0b013e318030c8dd

[59] Spiva L, Hart P, Pruner L, et al. Hearing the voices of newly licensed RNs: The transition to practice. The American Journal of Nursing. 2013; 113: 24-32. https://doi.org/10.1097/01.NAJ.00004 37108.76232 .20

[60] Tsai SL, Chai SK, Hsieh LF, et al. The use of virtual reality computer simulation in learning Port-A cath injection. Adv Health Sci Educ Theory Pract. 2008; 13: 71-87. https://doi.org/10.1007/s1 0459-006-9025-3

[61] Yoo MS, Park JH. Effect of case-based learning on the development of graduate nurses' problem-solving ability. Nurse Education Today. 2014; 34: 47-51. https://doi.org/10.1016/j.nedt.2013.02 .014

[62] Young P, Burke J. Evaluation of a multidisciplinary, simulation-based hospital residency program. Clinical Simulation in Nursing. 2010; 6: e45-e52. https://doi.org/10.1016/j.ecns.2009.08.005

[63] Soares CB, Yonekura T. Systematic review of theories: A tool to evaluate and analyze selected studies. Revista Da Escola de Enfermagem Da USP. 2011; 45: 1507-14. PMid:22241214 https: //doi.org/10.1590/S0080-62342011000600033

[64] Gaba DM. The future vision of simulation in healthcare. Simulation in Healthcare. 2007; 2: 126-35. https://doi .org/10.1097/01 .SIH.0000258411.38212.32 
[65] Hayden JK, Smiley RA, Alexander M, et al. The NCSBN national simulation study: A longitudinal, randomized, controlled study replacing clinical hours with simulation in prelicensure nursing education. National Council of State Boards of Nursing. 2014; 5 p.

[66] Aebersold M, Tschannen D. Simulation in nursing practice: the impact on patient care. Online J Issues Nurs. 2013; 18: 6.

[67] Radhakrishnan K, Roche JP, Cunningham H. Measuring clinical practice parameters with human patient simulation: A pilot study. Int $\mathrm{J}$ Nurs Educ Scholarsh. 2007; 4: Article 8. https ://doi .org/10. 2 202/1548-923X.1307

[68] Orique SB, Phillips LJ. The effectiveness of simulation on recognizing and managing clinical deterioration: Meta-analyses. Western Journal of Nursing Research. 2017; 1-28 p. https : //doi .org/10 $.1177 / 0193945917697224$

[69] Aggarwal R, Mytton OT, Derbrew M, et al. Training and simulation for patient safety. Qual Saf Health Care 2010; 19 Suppl 2: i34-43. https://doi.org/10.1136/qshc.2009.038562

[70] Makary MA, Daniel M. Medical error-The third leading cause of death in the US. BMJ. 2016; 353: i2139. PMid:27143499 https: //doi.org/10.1136/bmj.i2139

[71] McCarthy M. Efforts to improve US hospital safety stalled in 2014, report finds. BMJ: British Medical Journal (Online). 2015; 351 p. https://doi.org/10.1136/bmj.h6571

[72] Brakovich B, Bonham E. Solving the retention puzzle: Let's begin with nursing orientation. Nurse Leader. 2012; 10: 50-61. https: //doi.org/10.1016/j.mnl.2012.03.010

[73] Andel C, Davidow SL, Hollander M, et al. The economics of health care quality and medical errors. Journal of Health Care Finance. 2012; 39: 39. PMid:23155743

[74] Benner PE. From novice to expert: Excellence and power in clinical nursing practice. Commemorative Edition. Upper Saddle River, NJ: Prentice Hall: Pearson; 2001.

[75] NCBI. PubMed n.d. Available from: https://www.ncbi.nlm.n ih.gov/pubmed

[76] Berkeley. The top ten database search tips 2011. Available from: http://www.berkeleycitycollege.edu/wp/library /2011/04/04/databasesearchtips/

[77] Gopalakrishnan S, Ganeshkumar P. Systematic Reviews and Metaanalysis: Understanding the Best Evidence in Primary Healthcare. J Family Med Prim Care. 2013; 2: 9-14. https ://doi .org/10.410 3/2249-4863. 109934

[78] AHRQ. Advances in patient safety through simulation research 2016 Available from: https://grants.nih.gov/grants/guide/pa -files/PA-16-420.html 